\title{
Développement local, pauvreté et pratique contraceptive en Côte d'Ivoire
}

\author{
Édouard TALNAN \\ ENSEA \\ 08 BP 3, Abidjan 08 \\ Côte d'Ivoire
}

\author{
Patrice VIMARD \\ IRD \\ Université de Provence 151, Centre \\ St Charles, 3 pl. V. Hugo, 13331 \\ Marseille cedex 3, France
}

\section{Résumé}

La Côte d'Ivoire fait partie des pays d'Afrique subsaharienne n'ayant pas connu de politique de planification familiale jusqu'à la fin des années 1990. En revanche, une baisse de la fécondité peut être constatée depuis les années 1980, baisse qui s'articule avec une certaine progression de la prévalence contraceptive. Ce début de transition de la fécondité, qui se développe avec de fortes distinctions selon les milieux de résidence et les niveaux d'instruction, s'opère sous l'effet conjugué des facteurs de modernisation (urbanisation, développement de l'éducation et des services de santé, amélioration du statut de la femme, baisse de la mortalité des enfants, etc.). Mais cette transition s'inscrit également dans un contexte d'accroissement des contraintes économiques s'exerçant sur les ménages et les individus, de paupérisation et de précarité croissante de certaines couches de la population.

En utilisant les données de l'EDS de 1994 et un modèle d'analyse multi niveau, cet article étudie la pratique contraceptive en Côte d'Ivoire en vue d'identifier les facteurs individuels et contextuels explicatifs du recours aux méthodes de contraception, modernes d'un coté, naturelles et traditionnelles de l'autre. Les questions suivantes sont explorées dans le texte: les différences socio-économiques locales et individuelles, et notamment la pauvreté inégalement répartie des conditions de vie, influencent-elles l'usage de la contraception? Les différences d'accessibilité aux services de planification familiale, selon le secteur de résidence, contribuent-elles à la distinction des prévalences contraceptives? Quels sont les effets des interactions entre l'éducation et les variables des contextes régionaux et locaux sur la pratique contraceptive?

L'analyse multivariée, utilisée dans cette étude, permet de répondre à ces différentes questions parce qu'elle met en évidence la variation de la pratique contraceptive entre les milieux et les facteurs individuels et contextuels intervenant à l'intérieur de chacun d'eux. Concernant les facteurs individuels, l'importance de l'instruction de la femme, et dans une moindre mesure de son conjoint qui est généralement son chef de ménage, est démontrée ; concernant les facteurs du ménage et du contexte local, l'effet primordial du niveau de vie est mis en valeur. L'ensemble des résultats confirme l'association de la pauvreté humaine, caractérisée dans l'étude par un faible niveau d'instruction et des conditions de vie familiale et locale précaires, avec la faible pratique contraceptive. 
\{PASE

La Côte d'Ivoire fait partie des pays d'Afrique subsaharienne qui n'ont pas eu de réelle politique de planification familiale jusqu'à la fin des années 1990, tout en connaissant un début de baisse de leur fécondité et un relatif progrès de la contraception. Au cours des vingt années qui ont suivi son accession à l'indépendance en 1960, le pays a connu un taux de croissance démographique l'un des plus élevés au monde. Cette croissance de la population était due en partie à une hausse de la fécondité favorisée par la politique pro nataliste adoptée par le gouvernement. Celle-ci s'est traduite durant les années 1960 et 1970 par l'adoption du code pénal français interdisant l'avortement et la pratique contraceptive et l'adoption d'une politique d'allocation familiale qui consistait à verser des primes spéciales aux travailleurs du secteur moderne selon le nombre d'enfants vivants qu'ils avaient à leur charge. Au-delà de cette politique d'encouragement à la fécondité, le pouvoir politique ivoirien, incarné par la personne du président Félix Houphouët Boigny, avait également jugé nécessaire de favoriser l'immigration des populations étrangères pour combler le déficit de maind'œuvre afin d'assurer le développement économique du pays à travers l'exploitation des zones forestières. La politique de population existait en tant que politique de valorisation des ressources humaines et n'avait pour seuls objectifs que d'assurer l'autosuffisance alimentaire, la formation et l'éducation des populations, de fournir des emplois aux populations urbaines et $\mathrm{d}^{\prime}$ orienter les productions agricoles dans les zones rurales. C'est donc cette orientation politique qui était supposée, en l'absence totale d'une politique de planification familiale, déterminer l'évolution démographique. Et ce n'est qu'au début des 1990 que le pays a amorcé une politique de planification familiale, le gouvernement ayant reconnu, après la Conférence internationale sur la population et le développement (CIPD) tenue au Caire en septembre 1994, sous la pression des organisations internationales, la nécessité de développer des programmes pour offrir aux populations des services de qualité en matière de santé de la reproduction.

En parallèle à cette évolution politique, la population a amorcé une véritable transition de la fécondité. L'indice synthétique de fécondité est passé de 7,2 enfants par femme dans la période 1975-1980 à 5,2 enfants par femme lors de la période 1994-1998. Encore relativement élevé au niveau national, cet indicateur varie en fonction du secteur de résidence : la ville d'Abidjan ayant le niveau le plus bas avec 3,4 enfants par femme, suivie des autres centres urbains (4,9 enfants par femme) et des zones rurales où l'on dénombre en moyenne 6 enfants par femme. Les niveaux de fécondité diffèrent également selon le niveau d'instruction atteint par la femme : 2,3 enfants pour les femmes d'instruction secondaire ou supérieur, 4,7 enfants pour les femmes d'instruction primaire et 6,1 enfants pour les femmes sans instruction. Ainsi, la transition de la fécondité, amorcée vers le milieu des années 1970, s'accélère-t-elle au cours de ces vingt dernières années, sous l'effet conjugué des facteurs de modernisation tels que l'urbanisation, le développement de l'éducation et des services de santé, l'amélioration du 
Edouard Talman \& Patrice Vimard: Développement local, pauvreté et pratiq|PAGE \} contraceptive en Côte d'Ivoire

statut de la femme, la baisse de la mortalité des enfants, etc. (Vimard et al., 2002). Mais cette transition s'inscrit également dans un contexte d'accroissement des contraintes économiques s'exerçant sur les ménages et les individus, de paupérisation et de précarité croissante de certaines couches de la population et de développement des inégalités induit par la crise des années 1980, la baisse du pouvoir d'achat des populations et le désengagement de l'Etat du soutien aux services publics.

Ce début de diminution de la fécondité s'est articulé avec des progrès de la pratique contraceptive. En 1980 la prévalence contraceptive était de 0,6 \% pour les méthodes modernes et 3,2 \% pour les méthodes naturelles ou traditionnelles. Dix-huit ans plus tard, en 1998-99, cette prévalence contraceptive est de $9,8 \%$ pour les méthodes modernes et $10,9 \%$ pour les méthodes naturelles ou traditionnelles. L'utilisation de la contraception est plus élevée dans les groupes socio-économiques les plus favorisés et plus faibles dans les groupes démunis. Par exemple chez les femmes mariées, la prévalence contraceptive est de $40,3 \%$ chez les femmes d'instruction secondaire ou supérieur, et de $7,7 \%$ seulement pour les femmes sans instruction (INS et ORC Macro, 2001).

Dans la mesure où les progrès économiques et sociaux ont concerné de manière très inégale les différentes populations ivoiriennes et où les services de planification familiale ont été mis en place lentement et avec de fortes inégalités spatiales, les villes étant plus favorisées que les zones rurales, les régions forestières du sud l'étant davantage que celles de savane au nord (Anoh et al., 2005), on pourrait s'attendre à ce que leurs effets sur les comportements de fécondité se traduisent par une hétérogénéité entre les contextes locaux. Les évolutions de la contraception risquent, par conséquent, d'être différentes entre les régions et les localités à l'intérieur de ces régions, selon les capacités d'innovation technologique ou institutionnelle des populations, les opportunités économiques et foncières, les efforts réalisés pour améliorer le cadre de vie des populations, les possibilités d'échanges et la qualité des infrastructures permettant de communiquer avec les autres régions du pays et avec le monde.

Notre article vise par conséquent à identifier les facteurs explicatifs du recours aux méthodes contraceptives modernes, naturelles ou traditionnelles de en Côte d'Ivoire, et notamment de différencier ceux qui relèvent des caractéristiques individuelles des femmes de ceux qui concernent leur contexte local de vie. Les questions suivantes sont explorées dans le texte: les différences socio-économiques locales et individuelles, et notamment la pauvreté inégalement répartie des conditions de vie, influencent-elles les comportements de fécondité notamment le recours aux méthodes contraceptives ? Quelles sont, selon le secteur de résidence, les différences en 
\{PACGi\} Population Studies Vol. $21 n^{\circ} 1 /$ Etude de la population africaine vol. $21 n^{\circ} 2$

matière d'accessibilité aux services de planification familiale ? Comment le manque d'accès aux services de planification familiale et les conditions de vie dans le ménage peuvent-ils expliquer les différences en matière de pratique contraceptive en Côte d'Ivoire? Ces questions sont particulièrement importantes pour évaluer les efforts en matière de politique de population et orienter les programmes de planification familiale dans les années à venir, en fonction des niveaux et des types de pauvreté.

Les études sur la transition de la fécondité en Côte d'Ivoire ainsi que les approches méthodologiques jusque là utilisées nous ont semblé peu adaptées pour répondre à ces questions. C'est pourquoi nous avons opté pour une approche contextuelle susceptible de nous aider à mieux cerner les facteurs associés à l'utilisation des méthodes contraceptives. Notre attention s'est donc portée plus particulièrement sur les modèles multi niveaux dont la pertinence pour une analyse explicative des comportements de fécondité a été mise en évidence dans la littérature des vingt dernières années. L'utilisation de ces modèles doit nous permettre d'estimer l'influence différentielle de la disponibilité des services de planification familiale selon le niveau de capital social des groupes de population, mais aussi la relative conjugaison des niveaux de pauvreté (contextuel, familial et individuel) dans l'impossibilité pour certains individus d'accéder à la contraception moderne et à la pleine maîtrise de leur vie reproductive ${ }^{1}$.

\section{Sources de données et méthodes d'analyse}

\section{I.1. Présentation des sources de données}

Les données utilisées dans cette étude proviennent principalement de l'enquête démographique et de santé (EDS) conduite en 1994 par l'Institut national de la statistique, en collaboration avec Macro International2 ${ }^{2}$. Dans notre étude, seules les femmes en union au moment de l'enquête ont été prises en compte pour la simple raison, qu'avec la progression rapide du VIH/Sida, un nombre important de femmes n'étant pas en union utilise le condom avec leur partenaire en vue de se préserver contre cette pandémie, de sorte qu'il est difficile de distinguer celles utilisant le condom pour se protéger du VIH de celles l'utilisant pour éviter une grossesse. Sur les 246 grappes enquêtées, 238 pour lesquelles nous avons pu disposer de données complètes sur les infrastructures sociales et économiques ont été retenues, ce qui correspond à un effectif total de 5029 femmes mariées dont 2850 en milieu rural et 2179 en milieu urbain. Le fait d'omettre certaines grappes dans notre échantillon d'analyse n'a aucun effet sur les résultats.

Outre les données de l'enquête démographique de 1994, nous avons utilisé celles du Recensement général de la population et de l'habitat (RGPH) de 1998 qui a collecté des informations sur les communautés, notamment la présence de certaines infrastructures socio-économiques. Ces 
Edouard Talman \& Patrice Vimard: Développement local, pauvreté et pratiq|PAGE \} contraceptive en Côte d'Ivoire

informations ont permis de compléter les données communautaires de l'EDS de 1994. Un fichier «multi niveau » a donc été constitué à l'aide du progiciel SPSS (version 10.0) et MLWin (version 1.10) en combinant les informations sur les caractéristiques communautaires fournies par le recensement avec des données individuelles collectées dans le questionnaire individuel de l'EDS. Pour cela, nous avons considéré qu'une infrastructure existait dans la localité si sa réalisation était antérieure à 1994. Ceci nous permet d'évaluer le niveau de développement socio-économique de la localité et la disponibilité de services de planification familiale pour chaque localité rurale ou chaque quartier urbain; en effet, pour les besoins de l'analyse, nous distinguons le milieu urbain du milieu rural.

\section{I - 2. Définition des variables d'étude}

\section{Les variables dépendantes}

Notre variable dépendante principale est l'utilisation de la contraception par les femmes en union. Parce que nous avons des données récentes sur les caractéristiques contextuelles et individuelles des femmes, nous avons jugé plus pertinent de limiter notre analyse à l'utilisation d'une méthode contraceptive moderne, naturelle ou traditionnelle au moment de l'enquête. Ce choix répond aux critiques de DeGraff et al. (1997, cités par Schoumaker, 2001a) lorsqu'ils proposent de mettre les comportements récents en relation avec les conditions contextuelles récentes ou antérieures, même si on peut s'interroger sur le temps nécessaire pour que les changements intervenus au niveau du contexte puissent entraîner des modifications au niveau des comportements individuels (Bhattacharyya, 1989). L'un des problèmes liés à la variable "utilisation d'une méthode de contraception dans le passé », relevé par Alaudin (1983), est le fait que la variable dépendante peut être antérieure à certaines des variables explicatives, aussi nous n'avons pas retenu ce type de variables.

Les femmes qui utilisent les méthodes naturelles ou traditionnelles ont été également prises en compte dans cette analyse, parce qu'à défaut de pouvoir accéder aux méthodes modernes, l'utilisation de ces méthodes peut révéler l'efficacité des stratégies d'information, d'éducation et de communication sur l'espacement ou la limitation des naissances, et traduire la volonté des femmes de contrôler leur fécondité. Notre variable dépendante a donc deux composantes qui sont l'utilisation de la contraception moderne et l'utilisation des méthodes naturelles ou traditionnelles. Elles ont toutes deux un caractère dichotomique et prennent la modalité «oui=1» pour toutes les femmes ayant déclaré utiliser une méthode de contraception au moment de l'enquête et la modalité " non=0 » lorsque ce n'est pas le cas. 
\{PASE

Les variables déterminantes : caractéristiques individuelles, caractères du ménage et conditions de vie locales en termes d'accès aux infrastructures socio-économiques modernes

Pour décrire les conditions de vie des femmes de notre échantillon, nous avons utilisé trois types de variables choisis au niveau individuel et communautaire dans une perspective multi niveau. Ces variables permettent de prendre en compte les différences culturelles ainsi que les écarts socio-économiques, liés à l'acquisition d'un certain capital humain, et les disparités environnementales relatives à l'amélioration du cadre de vie. $\mathrm{Au}$ niveau individuel, les variables retenues concernent les caractéristiques démographiques et socio-économiques de chaque femme enquêtée: âge, ethnie, religion, milieu de socialisation ${ }^{3}$, instruction, secteur d'activité, expérience vécue à propos de la mortalité ou du confiage d'un enfant. Plusieurs études ont par exemple montré que les femmes mieux instruites ont aujourd'hui une meilleure chance de pratiquer la contraception moderne que leurs paires dont l'éducation est moins poussée, et qu'elles risquent par conséquent moins, surtout dans les endroits où les programmes de planification familiales sont efficaces, d'avoir des grossesses non désirées (Kalam et Khan, 1996). De même, il est établi dans la littérature que les femmes qui exercent une activité économique dans le secteur moderne ont une meilleure connaissance des méthodes de contraception et les utilisent plus que leurs homologues qui n'ont jamais travaillé dans ce secteur et qui sont dans le secteur agricole ou informel.

$\mathrm{Au}$ niveau de l'unité domestique, nous utilisons comme indices de conditions de vie des femmes le niveau d'instruction du chef de ménage, la structure familiale du ménage de résidence ${ }^{4}$, et le niveau de vie du ménage. Celui-ci est mesuré par un indicateur composite construit sur la base de variables relatives à la disposition d'un certain type de biens matériels (télévision, radio, frigidaire, voiture, etc.) et aux caractéristiques du logement (nature du plancher ou du sol, approvisionnement en eau, mode d'éclairage, type de toilettes utilisées) qui permet de distinguer les femmes selon qu'elles appartiennent à un ménage dont le standing de vie est élevé, moyen ou bas.

Par ailleurs, les analyses contextuelles des vingt dernières années ont montré que le développement communautaire et les institutions locales sont des facteurs importants de changement social au niveau de ces localités (De Jong et al., 1989; Bilsborrow et al., 1989; Schoumaker, 2001b). Ainsi, si l'attitude des femmes envers la planification familiale varie selon les caractéristiques socio-économiques individuelles ou familiales, elle est également influencée par différents facteurs exogènes à l'individu relevant aussi bien de l'environnement géographique que de la disponibilité ou de 
Edouard Talman \& Patrice Vimard: Développement local, pauvreté et pratiq|PAGE \} contraceptive en Côte d'Ivoire

l'accessibilité des services de planification familiale, et du groupe social et culturel auquel elles appartiennent (Kouamé et al, 2002). Si bien que, pour mieux comprendre la réalité de l'association entre les conditions de vie des femmes et leurs pratiques en matière de contraception, il nous est apparu important de prendre en compte le contexte social et économique dans lequel se trouve la femme au moment de l'enquête en vue d'estimer l'effet de certaines variables non individuelles pouvant être à l'origine des décisions en matière de pratique contraceptive. Dans le cadre de cette étude, trois groupes de variables ont été retenus pour caractériser le contexte dans lequel les décisions de recours aux méthodes contraceptives sont prises dans les ménages: les variables qui caractérisent le mieux le système de production de la communauté notamment le type d'agriculture d'exportation pratiquée principalement dans la localité, le niveau de développement social et économique de la localité et celles qui rendent compte des efforts du gouvernement et des autres institutions en matière de politique de planification familiale. En effet, l'une des caractéristiques des pays en développement est la persistance d'inégalités sociales dues en grande partie à une mauvaise répartition des ressources nécessaires pour assurer de meilleures conditions de vie aux populations (Vimard et al., 2002).

À cause de la crise économique et l'ajustement structurel, les investissements ont été globalement faibles et inégalement répartis en Côte d'Ivoire au cours des années 1990. Les conséquences sur les secteurs sociaux se sont notamment traduites par une réduction des ressources financières et une dégradation des structures existantes, qui démontrent l'incapacité actuelle de l'Etat à couvrir les besoins des populations en matière de santé et d'éducation. Selon une étude réalisée récemment par Mancini et al. (2003), les services de planification familiale existent dans seulement 4 régions sur les 19 que compte le pays.

C'est pour ces raisons que, pour caractériser le contexte socioéconomique local, nous avons considéré, pour cette étude, la principale culture d'exportation, le niveau de la mortalité des enfants de moins de cinq ans, la proportion d'adultes ayant été scolarisés et le pourcentage d'enfants de moins de 16 ans fréquentant l'école au moment de l'enquête. La technique de l'analyse factorielle a été utilisée pour construire un indicateur agrégé de développement socio-économique communautaire dans chaque secteur de résidence basé sur la présence d'un certain nombre $\mathrm{d}$ 'infrastructures sociales et économiques, qui, en ville comme dans les zones rurales, sont supposées être favorables à un accroissement de la pratique contraceptive. En milieu rural, cet indicateur est basé sur la proximité du village à un chef-lieu de sous-préfecture et la présence de certaines infrastructures socio-économiques telles que l'électrification, la source d'approvisionnement en eau, la voie d'accès à la localité, l'existence et la proximité d'un service de santé, le mode de collecte des ordures et 
\{PASE

d'évacuation des eaux usées et l'existence d'un certain nombre d'établissements ou de services (école primaire, gare routière, marché de vivres et marchandises, dépôt de médicaments).

Dans les zones urbaines, les variables suivantes ont été retenues pour la construction de cet indicateur: mode d'évacuation des eaux usées et des ordures, voie d'accès à la localité, statut administratif de la localité (Abidjan, chef-lieu de département, chef-lieu de sous-préfecture), proportion d'acteurs ruraux dans la localité, type de formation sanitaire existant dans la localité, année d'ouverture de cette formation sanitaire, et existence d'un certain nombre d'établissements (centre culturel, foyer féminin, centre social, équipement agricole). Compte tenu de la présence d'écoles et de structures sanitaires dans la plupart des villes ivoiriennes, la simple existence de ces deux services n'a pas été retenue pour la construction de cet indicateur en milieu urbain.

S'agissant des efforts politiques et institutionnels en matière de santé reproductive, nous avons utilisé comme variable explicative contextuelle l'accessibilité aux services de planification familiale dans la localité mesurée par la présence d'un agent ou d'un animateur de planification familiale, l'existence d'une campagne de planification familiale ou d'un centre de santé délivrant des services de planification familiale et les conditions pour y accéder. Ces variables sont disponibles dans le fichier communautaire de l'enquête démographique et de santé de 1994 et elles sont identifiées, dans la littérature, comme faisant partie des facteurs les plus pertinents, d'un point de vue contextuel, en étant susceptibles d'influencer significativement les attitudes et comportements individuels en matière de fécondité. Leurs effets sur la pratique contraceptive ont particulièrement été mis en évidence dans plusieurs pays à travers le monde à l'aide des enquêtes mondiales de fécondité (Casterline, 1985; Entwisle et al., 1986) et des enquêtes démographiques et de santé (Guilkey et Jayne, 1997 ; Nguyen et Nguyen, 2002). L'hypothèse à la base de cette relation étant que la présence de ces services influence les décisions et les comportements des femmes en matière de planification familiale de sorte que les femmes qui ont un accès facile à ces services sont davantage susceptibles d'y recourir pour limiter ou espacer leurs naissances que celles qui $\mathrm{n}^{\prime} \mathrm{y}$ ont pas facilement accès. Toutes ces variables ont été ensuite transformées en variables dichotomiques prenant la valeur 1 si le service en question existe dans la localité et 0 dans le cas contraire. Un indice composite d'accessibilité aux méthodes modernes de planification familiale, construit en faisant la somme de ces quatre variables indicatrices, permet de classer les communautés selon qu'elles ont un accès facile, moyen ou difficile à ces services 5 . 
Edouard Talman \& Patrice Vimard: Développement local, pauvreté et pratiq|PAGE \} contraceptive en Côte d'Ivoire

\section{I.3. Méthode d'analyse}

Nous avons effectué une analyse explicative, à l'aide du modèle de régression logistique multi niveau, en vue de cerner les facteurs contextuels et individuels qui déterminent le recours à la contraception par les femmes et le mécanisme par lequel les variables de contexte influencent la fécondité via les caractéristiques individuelles. Nous nous sommes limités à deux niveaux d'analyse : la femme et son ménage au premier niveau et le contexte local (du village ou du quartier) au second niveau.

\section{Les résultats}

\section{II.1. Facteurs individuels et contextuels de la pratique contraceptive}

Dans cette partie de notre analyse, nous tenterons de circonscrire, à partir d'une analyse de régression multiple, l'effet propre de chacune des caractéristiques décrivant les conditions de vie des femmes sur leurs comportements en matière de régulation de la fécondité. Pour chacune de ces méthodes contraceptives, deux modèles ont été estimés en plus du modèle vide ${ }^{6}$ (modèle 1 ). Le modèle 2 contient des variables explicatives relatives aux caractéristiques de la femme et de son ménage d'appartenance. Le modèle 3 intègre l'ensemble des variables contextuelles décrivant les caractéristiques du village ou du quartier de résidence de la femme en plus de l'ensemble des variables relatives aux individus et aux ménages introduites dans le modèle 2. Les coefficients de régression sont des Odd Ratio et sont interprétés comme étant des rapports de chance de l'utilisation de la contraception chez une catégorie de femmes quand on les compare aux femmes ayant la modalité de référence ${ }^{7}$.

\section{L'analyse multi niveau de la contraception en milieu urbain}

Les résultats du modèle vide (modèles a.1 et b.1 du tableau 1) montrent qu'en moyenne, $7,7 \%$ des femmes urbaines utilisent une méthode contraceptive moderne au moment de l'enquête et 10,7\% d'entre elles utilisent une méthode traditionnelle ou naturelle. Mais le niveau de la pratique contraceptive varie significativement $(p<0.01)$ en fonction des aires géographiques (correspondant aux grappes enquêtées) avec une variance contextuelle de 0,3 pour les méthodes modernes et 0,451 pour les méthodes naturelles ou traditionnelles. La prise en compte des caractéristiques individuelles de la femme annule la variation de la pratique contraceptive moderne entre les grappes (de 0,3 à 0,000) et réduit fortement, de 62,7\%, celle des méthodes naturelles ou traditionnelles (de 0,451 , elle passe à 0,169 dans le modèle 2). La nullité ou le caractère non significatif de la variance contextuelle dans les modèles 2 et 3 montre que la prise en compte des 
\{PASE

variables explicatives correspondantes contribue à une meilleure spécification de ces modèles. Autrement dit, l'inclusion d'autres variables explicatives n'apporterait pas d'éléments importants supplémentaires à l'explication de la variation de la pratique contraceptive entre les aires géographiques ou les quartiers.

Toutes choses égales par ailleurs, l'âge n'est pas un facteur statistiquement significatif de l'usage de la contraception (la seule relation significative concerne l'âge au carré et l'utilisation d'une méthode moderne, mais l'effet est très faible). Par contre, l'éducation, mesurée par le niveau d'instruction de la femme, est un facteur discriminant de l'utilisation des méthodes contraceptives $(p<0,01)$. Son effet sur l'utilisation des méthodes modernes est manifeste et conforme aux hypothèses émises dans la théorie de la transition de la fécondité en Afrique et dans d'autres régions du monde en développement. Avec un rapport de chance respectivement de 2,046 et 2,083 , il traduit le fait que les femmes qui ont atteint un niveau d'instruction élevé, équivalent au niveau primaire ou au niveau secondaire ou supérieure, sont davantage susceptibles d'utiliser une méthode contraceptive moderne. Elles ont également respectivement 1,711 et 3,380 fois plus de chance d'utiliser une méthode contraceptive traditionnelle ou naturelle lorsqu' on les compare aux femmes sans instruction. Il en est de même pour les femmes dont le chef de ménage est instruit au niveau primaire ou au niveau secondaire ou supérieur, qui ont respectivement 1,551 et 1,714 fois plus de chance d'utiliser une méthode traditionnelle ou naturelle $(\mathrm{P}<0,05)$. Mais les résultats de notre analyse montrent que contrairement à l'éducation de la femme, l'effet de l'instruction du chef de ménage sur la pratique contraceptive moderne n'est pas significatif au seuil de $5 \%$. Autrement dit, il n'y a aucune différence du point de vue de l'utilisation des méthodes contraceptives modernes entre les femmes dont le chef de ménage (qui est généralement le conjoint) est instruit et celles qui vivent dans des ménages dont le chef n'a aucun niveau d'instruction.

La décision pour une femme mariée vivant en ville de recourir à la contraception moderne dépend aussi du groupe ethnique auquel elle appartient. Ainsi, comparées aux femmes du groupe akan, si les femmes krou n'ont pas de comportements significativement différents vis-à-vis de ces méthodes, les analyses montrent que celles des deux groupes mandé, comme les femmes provenant des autres pays africains, ont des chances significativement plus faibles de pratiquer une contraception au moment de $l^{\prime}$ enquête $(p<0,01)$. Le niveau de vie du ménage $n^{\prime}$ influence pas de manière très significative la décision des femmes de recourir aux méthodes contraceptives modernes $(\mathrm{P}<0,1)$. Cependant, on note que les femmes qui ont un niveau de vie élevé ont plus de chance $(\mathrm{OR}=4,845)$ d'utiliser une méthode de contraception moderne par rapport à leurs homologues dont les conditions de vie dans le ménage sont précaires. De même, celles qui vivent dans des ménages dont les conditions de vie sont moyennes ont 2,565 fois plus de chance d'utiliser une méthode contraceptive moderne par rapport 


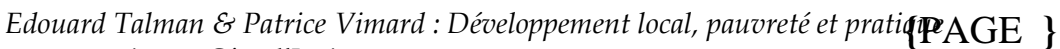
contraceptive en Côte d'Ivoire

aux femmes pauvres. Aucune différence significative, à moins de 5\%, n'est observée concernant l'usage des méthodes traditionnelles et naturelles.

L'introduction des variables contextuelles dans le modèle modifie légèrement les coefficients sans apporter un changement dans le sens de la relation entre les caractéristiques individuelles et la pratique contraceptive. Les estimations à partir du modèle a.3 montrent également que le niveau de développement socio-économique local, la proportion d'enfants ou d'adultes scolarisés dans le quartier sont des facteurs contextuels importants pour la pratique contraceptive chez les femmes citadines. On constate qu'au seuil de $1 \%(\mathrm{P}<0,01)$, le fait de vivre dans un quartier où la proportion de personnes ayant été scolarisées est supérieure ou égale à 50 \% entraîne une probabilité moins élevée chez la femme de déclarer l'utilisation d'une méthode contraceptive moderne $(\mathrm{OR}=0,493)$. Ce résultat, surprenant au premier abord, est en réalité normal car l'effet que peut avoir un milieu de forte instruction sur la pratique contraceptive est médiatisée par d'autres variables telles que l'instruction individuelle de la femme, celle de son conjoint, la scolarisation des enfants ou les conditions de vie des populations. En d'autres termes, le caractère négatif de l'effet de la variable relative au niveau global de scolarisation de la population sur la pratique contraceptive indique que si toutes les femmes avaient les mêmes caractéristiques individuelles et vivaient dans les mêmes conditions de développement, la propension à utiliser la contraception moderne serait moins élevée dans les quartiers où le niveau de la scolarisation est plus fort. La proportion d'enfants scolarisés a un effet significatif sur l'utilisation de la contraception moderne, avec un rapport de chance de 1,027 pour une augmentation de $1 \%$ de la proportion d'enfants scolarisés dans le quartier $(\mathrm{p}<0,01)$, comme de la contraception naturelle ou traditionnelle, avec un rapport de chance de 1,015 ( $\mathrm{p}<0,05)$.

Par contre le niveau de développement socio-économique local ne semble pas être un facteur statistiquement discriminant de l'usage de la contraception, même si on peut constater qu'à $P<0,1$, les femmes qui vivent dans des localités avec un niveau de développement moyen ont plus de chance d'utiliser la contraception moderne, avec un rapport de chance de 1,543. Il en est de même pour le niveau de la mortalité infantile qui n'a pas d'effet significatif sur la contraception en milieu urbain. 
\{PAEFcal\} Population Studies Vol. $21 n^{\circ} 1 /$ Etude de la population africaine vol. $21 n^{\circ} 2$

TABLEAU 1 : LES FACTEURS DE LA PRATIQUE CONTRACEPTIVE CHEZ LES FEMMES MARIEES EN MILIEU URBAIN. COTE D'IVOIRE. EDS 1994

\begin{tabular}{|c|c|c|c|c|c|c|}
\hline \multirow[t]{2}{*}{ Variables explicatives } & \multicolumn{3}{|c|}{ Méthodes modernes } & \multicolumn{3}{|c|}{$\begin{array}{l}\text { Méthodes naturelles } \\
\text { ou traditionnelles }\end{array}$} \\
\hline & $\begin{array}{l}\text { Modèle } \\
\text { a.1 }\end{array}$ & $\begin{array}{l}\text { Modèle } \\
\text { a.2 } \\
\end{array}$ & $\begin{array}{l}\text { Modèle } \\
\text { a.3 } \\
\end{array}$ & $\begin{array}{c}\text { Modèle } \\
\text { b.1 }\end{array}$ & $\begin{array}{c}\text { Modèle } \\
\text { b.2 }\end{array}$ & $\begin{array}{c}\text { Modèle } \\
\text { b. } 3\end{array}$ \\
\hline Constante a $^{a}$ & $7,7^{* * *}$ & $0,08^{* * *}$ & 0,037 *** & $10,7^{* * *}$ & $33,9 \mathrm{~ns}$ & $28,7 \mathrm{~ns}$ \\
\hline Variables individuelles & & & & & & \\
\hline $\begin{array}{l}\text { Age de la femme } \\
\text { Age au carré }\end{array}$ & - & $\begin{array}{l}1,153 \mathrm{~ns} \\
0,998 * *\end{array}$ & $\begin{array}{l}1,137 \mathrm{~ns} \\
0,998^{* *}\end{array}$ & - & $\begin{array}{l}0,943 \mathrm{~ns} \\
1,001 \mathrm{~ns}\end{array}$ & $\begin{array}{l}0,937 \mathrm{~ns} \\
1,001 \mathrm{~ns}\end{array}$ \\
\hline $\begin{array}{l}\text { Milieu de socialisation (Villages) } \\
\text { Grandes villes } \\
\text { Petites villes }\end{array}$ & - & $\begin{array}{c}1,584 * * \\
1,357 \mathrm{~ns}\end{array}$ & $\begin{array}{l}1,526 \mathrm{~ns} \\
1,354 \mathrm{~ns}\end{array}$ & - & $\begin{array}{l}0,968 \mathrm{~ns} \\
0,894 \mathrm{~ns}\end{array}$ & $\begin{array}{l}0,975 \mathrm{~ns} \\
0,895 \mathrm{~ns}\end{array}$ \\
\hline $\begin{array}{l}\text { Religion (Musulmane) } \\
\text { Chrétienne } \\
\text { Autre religion/sans religion }\end{array}$ & - & $\begin{array}{l}0,600 \mathrm{~ns} \\
0,837 \mathrm{~ns}\end{array}$ & $\begin{array}{l}0,572 \mathrm{~ns} \\
0,823 \mathrm{~ns}\end{array}$ & - & $\begin{array}{l}1,320 \mathrm{~ns} \\
0,854 \mathrm{~ns}\end{array}$ & $\begin{array}{l}1,245 \mathrm{~ns} \\
0,829 \mathrm{~ns}\end{array}$ \\
\hline $\begin{array}{l}\text { Ethnie de la femme (Akan) } \\
\text { Krou } \\
\text { Mandé du Nord et Gur } \\
\text { Mandé du Sud } \\
\text { Autres africains } \\
\end{array}$ & $\begin{array}{l}- \\
- \\
- \\
-\end{array}$ & $\begin{array}{c}0,660 \mathrm{~ns} \\
0,310 * * * \\
0,506 * * \\
0,311^{* * *}\end{array}$ & $\begin{array}{l}0,679 \mathrm{~ns} \\
0,306 * * * \\
0,495 * * \\
0,319 * * * \\
\end{array}$ & $\begin{array}{l}- \\
- \\
- \\
-\end{array}$ & $\begin{array}{c}0,678 \mathrm{~ns} \\
0,540 * * \\
0,695 \mathrm{~ns} \\
0,327 * * *\end{array}$ & $\begin{array}{l}0,684 \mathrm{~ns} \\
0,562 * * \\
0,695 \mathrm{~ns} \\
0,332 * * *\end{array}$ \\
\hline $\begin{array}{l}\text { Confiage des enfants (Au moins un enfant confié) } \\
\text { Aucun enfant confié }\end{array}$ & - & $0,640 * *$ & $0,639 * *$ & - & 0,969 ns & $0,966 \mathrm{~ns}$ \\
\hline $\begin{array}{l}\text { Instruction (Sans instruction) } \\
\text { Primaire } \\
\text { Secondaire \& supérieure }\end{array}$ & $\begin{array}{l}- \\
-\end{array}$ & $\begin{array}{l}2,046 * * * \\
2,083 * * *\end{array}$ & $\begin{array}{l}2,026 * * * \\
2,040 * * *\end{array}$ & $\begin{array}{l}- \\
-\end{array}$ & $\begin{array}{l}1,711 * * * \\
3,380 * * *\end{array}$ & $\begin{array}{l}1,711^{* * *} \\
3,294 * *\end{array}$ \\
\hline
\end{tabular}




\begin{tabular}{|c|c|c|c|c|c|c|}
\hline $\begin{array}{l}\text { Secteur d'activité (Agriculture) } \\
\text { Sans activité } \\
\text { Formel } \\
\text { Informel }\end{array}$ & $\begin{array}{l}- \\
- \\
-\end{array}$ & $\begin{array}{l}2,392 \mathrm{~ns} \\
2,125 \mathrm{~ns} \\
3,732 *\end{array}$ & $\begin{array}{c}2,333 \mathrm{~ns} \\
2,014 \mathrm{~ns} \\
3,554 *\end{array}$ & $\begin{array}{l}- \\
- \\
-\end{array}$ & $\begin{array}{l}0,901 \mathrm{~ns} \\
0,954 \mathrm{~ns} \\
1,004 \mathrm{~ns}\end{array}$ & $\begin{array}{l}0,920 \mathrm{~ns} \\
0,966 \mathrm{~ns} \\
1,014 \mathrm{~ns}\end{array}$ \\
\hline $\begin{array}{l}\text { Instruction } d u C M \text { (Sans instruction) } \\
\text { Primaire } \\
\text { Secondaire \& + }\end{array}$ & $\begin{array}{l}- \\
-\end{array}$ & $\begin{array}{l}0,841 \mathrm{~ns} \\
1,330 \mathrm{~ns}\end{array}$ & $\begin{array}{l}0,883 \mathrm{~ns} \\
1,311 \mathrm{~ns}\end{array}$ & $\begin{array}{l}- \\
-\end{array}$ & $\begin{array}{l}1,551 * * \\
1,714 * *\end{array}$ & $\begin{array}{l}1,549 * * \\
1,684 * *\end{array}$ \\
\hline $\begin{array}{l}\text { Structure du ménage (Sans famille nucléaire) } \\
\text { Nucléaire monogame et polygame } \\
\text { Elargie monogame } \\
\text { Elargie polygame }\end{array}$ & $\begin{array}{l}- \\
- \\
-\end{array}$ & $\begin{array}{l}1,044 \mathrm{~ns} \\
1,652 \mathrm{~ns} \\
1,435 \mathrm{~ns}\end{array}$ & $\begin{array}{l}1,013 \mathrm{~ns} \\
1,642 \mathrm{~ns} \\
1,507 \mathrm{~ns}\end{array}$ & $\begin{array}{l}- \\
- \\
-\end{array}$ & $\begin{array}{l}0,567 * * \\
0,668 * * \\
0,904 \mathrm{~ns}\end{array}$ & $\begin{array}{l}0,559 * * \\
0,660 * * \\
0,923 \mathrm{~ns}\end{array}$ \\
\hline $\begin{array}{l}\text { Niveau de vie du ménage (Bas) } \\
\text { Elevé } \\
\text { Moyen }\end{array}$ & $\begin{array}{l}- \\
-\end{array}$ & $\begin{array}{l}4,845^{* * *} \\
2,565 \mathrm{~ns}\end{array}$ & $\begin{array}{l}4,212 * * * \\
2,368 \mathrm{~ns}\end{array}$ & $\begin{array}{l}- \\
-\end{array}$ & $\begin{array}{l}0,904 \mathrm{~ns} \\
0,741 \mathrm{~ns}\end{array}$ & $\begin{array}{l}0,878 \mathrm{~ns} \\
0,745 \mathrm{~ns}\end{array}$ \\
\hline \multicolumn{7}{|l|}{ Variables liées au contexte local } \\
\hline $\begin{array}{l}\text { Niveau de développement local (Bas) } \\
\text { Elevé } \\
\text { Moyen }\end{array}$ & - & - & $\begin{array}{l}1,539 \mathrm{~ns} \\
1,543 \mathrm{~ns}\end{array}$ & - & - & $\begin{array}{l}0,996 \mathrm{~ns} \\
0,784 \mathrm{~ns}\end{array}$ \\
\hline $\begin{array}{l}\text { Pourcentage de personnes scolarisées ( }<50 \%) \\
\geq 50 \%\end{array}$ & - & - & 0,493 ** & - & - & $0,786 \mathrm{~ns}$ \\
\hline $\begin{array}{l}\text { Mortalité des enfants }(<125 \text { pour } 1000) \\
\geq 125 \text { pour } 1000\end{array}$ & - & - & $1,081 \mathrm{~ns}$ & - & - & $0,946 \mathrm{~ns}$ \\
\hline \% d'enfants scolarisés dans le quartier & - & - & $1,027^{* * *}$ & - & - & 1,015 ** \\
\hline $\begin{array}{l}\text { Variance au niveau des grappes }\left(\sigma_{v}^{2}\right) \\
\text { Variance au niveau des individus }\left(\sigma_{e}^{2}\right)\end{array}$ & $\begin{array}{l}0,300^{* *} \\
1,000^{* * *}\end{array}$ & $\begin{array}{c}0,000 \\
1,000 * * *\end{array}$ & \begin{tabular}{|l}
0,000 \\
$1,000 * * *$
\end{tabular} & $\begin{array}{l}0,451^{* * *} \\
1,000^{* * *}\end{array}$ & $\begin{array}{l}0,169 \mathrm{~ns} \\
1,000 * * *\end{array}$ & $\begin{array}{l}0,158 \mathrm{~ns} \\
1,000 * * *\end{array}$ \\
\hline
\end{tabular}


\{PASE

\section{L'analyse multi niveau de la contraception en milieu rural}

En milieu rural, le pourcentage de femmes utilisatrices de méthodes contraceptives est de $2,17 \%$ pour les méthodes modernes contre $4,9 \%$ pour les méthodes traditionnelles ou naturelles (tableau 2, modèles a.1 et b.1). Dans l'ensemble, il n'y a aucune variation significative entre les villages du point de vue de l'utilisation moyenne des méthodes modernes (la variance contextuelle n'est pas significative au seuil de $5 \%$ ). Parmi les variables explicatives individuelles choisies pour tester le modèle 2, seules l'instruction de la femme, son milieu de socialisation et la structure familiale du ménage apparaissent comme des facteurs significatifs de la pratique des méthodes contraceptives modernes.

Comparées aux femmes non instruites, celles qui ont atteint le niveau primaire ont 2,765 fois plus de chance de déclarer l'utilisation d'une méthode contraceptive moderne, et celles de niveau d'instruction supérieur ou secondaire, 5,296 fois plus de chance. Cela confirme l'hypothèse selon laquelle l'éducation de la femme favorise la pratique contraceptive dans les zones rurales $(\mathrm{P}<0,01)$. Les femmes qui ont grandi dans une ville secondaire ont plus de chance de déclarer l'utilisation d'une méthode contraceptive moderne $(\mathrm{OR}=2,016$ avec $\mathrm{P}<0,05)$. Par contre, celles qui ont été socialisées dans une grande ville n'ont pas d'attitude significativement différente vis-àvis de ces méthodes lorsqu'on les compare aux femmes qui ont grandi au village. Le fait de vivre dans un ménage dont la structure familiale est élargie monogame ou polygame augmente les chances de recourir à ces méthodes (le rapport de chance étant respectivement de 3,939 et 3,714 avec $\mathrm{p}<0,05)$. La prise en compte des variables explicatives contextuelles, comme le niveau de développement social et économique du village, la principale culture d'exportation pratiquée dans le village, le pourcentage d'enfants qui y sont scolarisés, le niveau de la scolarisation des adultes ou le quotient de mortalité des enfants de moins de cinq ans ne modifient pas significativement le sens de la relation obtenue précédemment. Et aucune de ces variables contextuelles n'est associée significativement à la pratique contraceptive moderne.

S'agissant de l'utilisation des méthodes naturelles ou traditionnelles, le modèle vide (modèle b.1) montre des différences significatives entre les villages qui se traduisent par une variance contextuelle de $0,643(p<0.01)$. En considérant les seules caractéristiques individuelles de la femme ainsi que celles relatives à son ménage de résidence (modèle b.2), on s'aperçoit que l'ethnie, l'éducation de la femme, le secteur d'activité, l'instruction du chef de ménage et la structure familiale du ménage de résidence sont les principaux facteurs responsables des différences d'utilisation des méthodes contraceptives naturelles ou traditionnelles. En effet, par rapport aux femmes akan, toutes les femmes des autres groupes ethniques ont moins de chance de déclarer la pratique d'une méthode contraceptive naturelle ou traditionnelle au moment de l'enquête avec une différente plus importante et très significative chez les femmes ressortissantes des autres pays africains $(\mathrm{P}<0,01)$. Avec un rapport de chance de 0,216 , elles sont nettement moins 


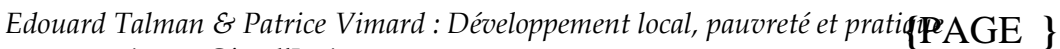
contraceptive en Côte d'Ivoire

susceptibles de déclarer l'utilisation d'une méthode contraceptive traditionnelle ou naturelle. En réalité, il s'agit de femmes non instruites en majorité épouses de travailleurs burkinabés ou maliens qui ne fréquentent généralement pas les centres de santé et sont moins portées vers l'adoption de stratégies visant à contrôler leur fécondité.

Comme dans les zones urbaines, les femmes instruites ont de meilleures chances d'utiliser les méthodes contraceptives naturelles ou traditionnelles par rapport à celles qui n'ont aucun niveau d'instruction. Le rapport de chance est de 2,930 pour le niveau primaire et de 4,332 pour le niveau secondaire ou supérieur $(p<0,01)$. Les femmes agricultrices ont moins de chance d'utiliser les méthodes contraceptives naturelles ou traditionnelles par rapport aux femmes qui exercent dans les secteurs modernes formels ou informels $(\mathrm{OR}=0,495$ avec $\mathrm{p}<0,01)$. La structure familiale du ménage de résidence de la femme au moment de l'enquête ainsi que l'éducation du chef sont également des facteurs associés à l'utilisation des méthodes contraceptives naturelles ou traditionnelles en milieu rural. En particulier, les femmes appartenant aux ménages nucléaires utilisent moins ces méthodes que celles dont le ménage est sans famille nucléaire et généralement composé de famille monoparentale ou d'un chef de ménage seul sans enfants $(\mathrm{OR}=0,520$ avec $\mathrm{p}<0,05)$. En milieu rural ivoirien, le fait de vivre dans un ménage dont le niveau d'instruction du chef est élevé accroît la probabilité de recourir aux méthodes contraceptives naturelles ou traditionnelles. C'est ainsi que, par rapport aux femmes résidant dans des ménages dont le chef est sans instruction, celles qui vivent dans des ménages dont le niveau d'instruction du chef est le primaire ont 2,109 fois plus de chance de faire usage de ces méthodes au moment de l'enquête ; ce rapport de chance est de 3,050 quand cette instruction atteint le niveau secondaire ou supérieur $(\mathrm{P}<0,01)$.

Dans le modèle 3 , on note que le niveau de développement économique et social du village $n^{\prime}$ influence pas le niveau de la pratique contraceptive naturelle ou traditionnelle chez les femmes rurales, même si les femmes qui vivent dans un village dont le niveau de développement est moyen ont 1,735 fois plus de chance d'utiliser ces méthodes $(p<0,1)$ par rapport aux femmes des villages défavorisés. Par contre, une variable contextuelle ayant un lien étroit avec la pratique des méthodes traditionnelles ou naturelles est le niveau de la mortalité des enfants de moins de cinq ans : une mortalité des enfants supérieure à 125 pour mille suscite chez les femmes un risque moindre de l'ordre de 0,535 d'utiliser une méthode contraceptive traditionnelle ou naturelle $(p<0,01)$. 
\{PAEEcan Population Studies Vol. $21 n^{\circ} 1 /$ Etude de la population africaine vol. $21 n^{\circ} 2$

TABLEAU 2 : LES FACTEURS DE LA PRATIQUE CONTRACEPTIVE CHEZ LES FEMMES MARIEES EN MILIEU RURAL. COTE D'IVOIRE. EDS 1994

\begin{tabular}{|c|c|c|c|c|c|c|}
\hline \multirow{2}{*}{ Variables explicatives } & \multicolumn{3}{|c|}{ Méthodes modernes } & \multicolumn{3}{|c|}{ Méthodes naturelles ou traditionnelles } \\
\hline & $\begin{array}{l}\text { Modèle } \\
\text { a.1 }\end{array}$ & $\begin{array}{l}\text { Modèle } \\
\text { a.2 }\end{array}$ & $\begin{array}{l}\text { Modèle } \\
\text { a.3 }\end{array}$ & $\begin{array}{c}\text { Modèle } \\
\text { b.1 }\end{array}$ & $\begin{array}{l}\text { Modèle } \\
\text { b.2 }\end{array}$ & $\begin{array}{c}\text { Modèle } \\
\text { b. } 3\end{array}$ \\
\hline Constante $^{a}$ & $2,17 * * *$ & $0,311^{* * *}$ & $0,184^{* * *}$ & $4,9 * * *$ & $0,801 * * *$ & $0,486^{* * *}$ \\
\hline Variables individuelles & & & & & & \\
\hline $\begin{array}{l}\text { Age de la femme } \\
\text { Age au carré }\end{array}$ & $\begin{array}{l}- \\
-\end{array}$ & $\begin{array}{l}1,021 \mathrm{~ns} \\
1,000 \mathrm{~ns}\end{array}$ & $\begin{array}{l}1,017 \mathrm{~ns} \\
1,000 \mathrm{~ns}\end{array}$ & - & $\begin{array}{l}1,202^{* *} \\
0,997 \mathrm{~ns}\end{array}$ & $\begin{array}{r}1,218 * * * \\
0,997 \mathrm{~ns}\end{array}$ \\
\hline $\begin{array}{l}\text { Milieu de socialisation (Villages) } \\
\text { Grandes villes } \\
\text { Petites villes }\end{array}$ & $\begin{array}{l}- \\
-\end{array}$ & $\begin{array}{l}1,323 \text { ns } \\
2,016 \text { ** }\end{array}$ & $\begin{array}{l}1,290 \mathrm{~ns} \\
2,015 * *\end{array}$ & $\begin{array}{l}- \\
-\end{array}$ & $\begin{array}{l}0,974 \mathrm{~ns} \\
0,807 \mathrm{~ns}\end{array}$ & $\begin{array}{c}0,961 \mathrm{~ns} \\
0,862 \mathrm{~ns}\end{array}$ \\
\hline $\begin{array}{l}\text { Ethnie de la femme (Akan) } \\
\text { Krou } \\
\text { Mandé du Nord et Gur } \\
\text { Mandé du Sud } \\
\text { Autres africains } \\
\end{array}$ & $\begin{array}{l}- \\
- \\
- \\
-\end{array}$ & $\begin{array}{l}2,106 * * \\
0,807 \mathrm{~ns} \\
0,954 \mathrm{~ns} \\
1,069 \mathrm{~ns}\end{array}$ & $\begin{array}{l}1,956 \mathrm{~ns} \\
1,039 \mathrm{~ns} \\
1,133 \mathrm{~ns} \\
1,234 \mathrm{~ns}\end{array}$ & $\begin{array}{l}- \\
- \\
- \\
-\end{array}$ & $\begin{array}{c}0,563 \text { ns } \\
0,495 * * \\
0,448 * * \\
0,216 * * *\end{array}$ & $\begin{array}{cc}0,729 & \mathrm{~ns} \\
0,787 & \mathrm{~ns} \\
0,683 & \mathrm{~ns} \\
0,258 * * * \\
\end{array}$ \\
\hline $\begin{array}{l}\text { Instruction (Sans instruction) } \\
\text { Primaire } \\
\text { Secondaire \& supérieure }\end{array}$ & $\begin{array}{l}- \\
-\end{array}$ & $\begin{array}{l}2,765 * * * \\
5,296 * * *\end{array}$ & $\begin{array}{l}2,596 * * * \\
4,711 * * *\end{array}$ & $\begin{array}{l}- \\
-\end{array}$ & $\begin{array}{l}2,930 * * * \\
4,332 * * *\end{array}$ & $\begin{array}{l}2,989 * * * \\
4,455 * * *\end{array}$ \\
\hline $\begin{array}{l}\text { Secteur d'activité (Formel/informel) } \\
\text { Sans activité } \\
\text { Agriculture }\end{array}$ & $\begin{array}{l}- \\
-\end{array}$ & $\begin{array}{c}0,478 \mathrm{~ns} \\
0,430 * * *\end{array}$ & $\begin{array}{l}0,505 \mathrm{~ns} \\
0,469\end{array}$ & $\begin{array}{l}- \\
-\end{array}$ & $\begin{array}{l}1,055 \mathrm{~ns} \\
0,495 * * *\end{array}$ & $\begin{array}{c}1,053 \mathrm{~ns} \\
0,490 * * * \\
\end{array}$ \\
\hline $\begin{array}{l}\text { Instruction du CM (Sans instruction) } \\
\text { Primaire } \\
\text { Secondaire \& supérieure }\end{array}$ & - & $\begin{array}{l}1,096 \mathrm{~ns} \\
1,570 \mathrm{~ns}\end{array}$ & $\begin{array}{l}1,032 \mathrm{~ns} \\
1,565 \mathrm{~ns}\end{array}$ & $\begin{array}{l}- \\
-\end{array}$ & $\begin{array}{l}2,109 * * * \\
3,050 * * * \\
\end{array}$ & $\begin{array}{l}2,100 * * * \\
3,196 * * *\end{array}$ \\
\hline
\end{tabular}




\begin{tabular}{|c|c|c|c|c|c|c|}
\hline $\begin{array}{l}\text { Structure du ménage (Sans famille nucléaire) } \\
\text { Nucléaire monogame et polygame } \\
\text { Elargie monogame } \\
\text { Elargie polygame }\end{array}$ & $\begin{array}{l}- \\
- \\
-\end{array}$ & $\begin{array}{l}0,585 \mathrm{~ns} \\
3,939 * * \\
3,714 * *\end{array}$ & $\begin{array}{l}0,595 \mathrm{~ns} \\
3,995 * * \\
3,732 * *\end{array}$ & $\begin{array}{l}- \\
- \\
-\end{array}$ & $\begin{array}{l}0,520 * * \\
0,779 \text { ns } \\
0,696 \text { ns }\end{array}$ & $\begin{array}{l}0,571 * * \\
0,837 \text { ns } \\
0,699 \text { ns }\end{array}$ \\
\hline $\begin{array}{l}\text { Niveau de vie du ménage (bas) } \\
\text { Elevé } \\
\text { Moyen }\end{array}$ & - & $\begin{array}{l}1,578 \mathrm{~ns} \\
0,573 \mathrm{~ns}\end{array}$ & $\begin{array}{l}1,336 \mathrm{~ns} \\
0,559 \mathrm{~ns}\end{array}$ & - & $\begin{array}{c}0,887 \mathrm{~ns} \\
0,724 \mathrm{~ns}\end{array}$ & $\begin{array}{l}0,935 \mathrm{~ns} \\
0,706 \mathrm{~ns} \\
\end{array}$ \\
\hline \multicolumn{7}{|l|}{ Variables liées au contexte local } \\
\hline $\begin{array}{l}\text { Produits agricoles d'exportation (café/cacao) } \\
\text { Coton/anacarde }\end{array}$ & - & - & $0,740 \mathrm{~ns}$ & - & - & $0,572 \mathrm{~ns}$ \\
\hline $\begin{array}{l}\text { Niveau de développement local (Bas) } \\
\text { Elevé } \\
\text { Moyen }\end{array}$ & - & - & $\begin{array}{l}1,742 \mathrm{~ns} \\
0,996 \mathrm{~ns}\end{array}$ & - & - & $\begin{array}{l}0,966 \mathrm{~ns} \\
1,735 * *\end{array}$ \\
\hline $\begin{array}{l}\text { Pourcentage de personnes scolarisées <25\%) } \\
\geq 25 \%\end{array}$ & - & - & $0,924 \mathrm{~ns}$ & - & - & $0,864 \mathrm{~ns}$ \\
\hline $\begin{array}{l}\text { Mortalité des enfants }(<125 \text { p.1000) } \\
\geq 125 \text { p.1000 }\end{array}$ & - & - & $0,800 \mathrm{~ns}$ & - & - & $0,535 * * *$ \\
\hline \% d'enfants scolarisés dans le village & - & - & $1,020 \mathrm{~ns}$ & - & - & $1,006 \mathrm{~ns}$ \\
\hline $\begin{array}{l}\text { Variance au niveau des grappes }\left(\sigma_{v}^{2}\right) \\
\text { Variance au niveau des individus }\left(\sigma_{\mathrm{e}}^{2}\right)\end{array}$ & $\begin{array}{l}0,294 \text { ns } \\
1,000 * * *\end{array}$ & $\begin{array}{l}0,000 \\
1,000 * * *\end{array}$ & $\begin{array}{l}0,000 \\
1,000 * * *\end{array}$ & $\begin{array}{l}0,643^{* * *} \\
1,000^{* * *}\end{array}$ & $\begin{array}{c}0,385^{* *} \\
1,000^{* * *}\end{array}$ & $\begin{array}{l}0,206 \mathrm{~ns} \\
1,000 * * *\end{array}$ \\
\hline
\end{tabular}

*** Significatif à $\mathrm{p}<0,01 \quad$ ** Significatif à $\mathrm{p}<0,05 \quad$ ns Non significatif à $\mathrm{p}<0,1$

() Modalité de référence

a : Exprimée en \% de femmes mariées utilisant une méthode de contraception dans l'ensemble de la population étudiée. 
\{PASÉa\} Population Studies Vol. $21 n^{\circ} 1 /$ Etude de la population africaine vol. $21 n^{\circ} 2$

\section{II.2. Instruction, développement local et pratique contraceptive}

L'éducation, en particulier celle de la femme, est apparue comme le principal facteur responsable de l'utilisation de la contraception en Côte $\mathrm{d}^{\prime}$ Ivoire, en milieu urbain comme en milieu rural. Or, cette variable peut interagir avec les caractéristiques globales ou contextuelles. En clair, les bénéfices liés à la mise en place d'infrastructures sociales et sanitaires et à la réalisation de projets de développement, exprimés en termes démographiques, seraient différents selon le niveau d'instruction des femmes. Les résultats obtenus dans plusieurs pays du monde en développement ont en effet confirmé ce résultat en montrant que les investissements consentis pour le bien-être des populations profitent en général moins aux populations analphabètes qu'à celles qui ont déjà atteint un niveau élevé d'instruction. En Côte d'Ivoire et au Ghana par exemple, une étude de Benefo et Shultz (1996), basée sur les données des enquêtes permanentes auprès des ménages réalisées au cours des années 1980, a montré que les programmes de développement profitaient différemment aux femmes en fonction de leur niveau d'éducation. Au Ghana, cette étude a montré que la disponibilité d'une source d'approvisionnement en eau potable contribue plus à réduire la mortalité des enfants chez les femmes instruites par rapport aux femmes non instruites. De même, l'éducation de la femme a un effet réducteur plus fort sur la mortalité des enfants en milieu rural qu'en milieu urbain. En Côte d'Ivoire, la même étude a montré que dans les communautés où il existe des latrines aménagées, l'effet de l'instruction sur la mortalité des enfants est plus fort que dans les autres communautés. Elle a donc conclu que l'éducation de la femme était un complément indispensable à des programmes de développement.

Cette approche peut être utilisée pour analyser la relation entre l'instruction de la femme et la contraception en fonction du contexte local. Dans ce cas, l'hypothèse serait que dans les quartiers ou les villages où existent des infrastructures et des services socio sanitaires ou des programmes de développement visant à offrir de meilleures conditions de vie aux populations, les femmes instruites seraient plus disposées à utiliser une contraception pour contrôler leur fécondité par rapport aux femmes non instruites. Cependant, l'existence d'un centre ou d'une campagne de sensibilisation en faveur de la planification familiale tendrait à réduire les différences induites par l'éducation des femmes en ce qui concerne l'utilisation des méthodes contraceptives. Par exemple, là où l'accès aux méthodes contraceptives est facile pour toutes les couches sociales, la différence entre la prévalence contraceptive des femmes instruites et celle mesurée chez les femmes non instruites ne serait pas significative (Ainsworth et al., 1996).

Pour tester la validité de cette hypothèse, nous avons construit dans cette dernière partie de l'article un modèle d'utilisation de la contraception dans lequel sont considérées comme variables explicatives le niveau d'instruction de la femme que nous mettons en interaction avec chacune des caractéristiques décrivant les conditions de vie dans la localité de résidence 
Edouard Talman \& Patrice Vimard: Développement local, pauvreté et pratiq|PAGE \} contraceptive en Côte d'Ivoire

de la femme, à savoir le secteur géographique de cette localité (forêt ou savane), le niveau de développement social et économique du contexte local, l'existence et le nombre d'établissements délivrant des services de planification familiale, l'existence d'une campagne de planification familiale, le niveau de la mortalité des enfants. Nous considérons comme variables dépendantes l'utilisation d'une méthode contraceptive au moment de l'enquête avec une distinction selon le type de méthode. Les variables originales utilisées pour créer les variables d'interaction (instruction de la femme, niveau de développement, mortalité des enfants, etc.) ne sont pas incluses dans le modèle compte tenu de leurs fortes corrélations avec les variables d'interaction. Toutes les autres variables (âge, lieu de socialisation, religion, etc.) sont incluses dans le modèle comme des variables de contrôle (tableau 3).

Les résultats obtenus montrent qu'en milieu urbain, il y a un effet d'interaction entre l'instruction de la femme et le secteur géographique de résidence. L'effet de cette interaction se manifeste par le fait que les femmes instruites dans les zones de savane ont plus de chance de pratiquer la contraception que celles résidant à Abidjan ou dans les zones forestières dotés du même niveau d'instruction: cet effet est significatif pour les méthodes modernes (1,176 fois plus de chance) comme pour les méthodes naturelles ou traditionnelles (1,102 fois plus de chance) $(\mathrm{P}<0,05)$. Par contre, les autres variables d'interaction n'ont pas d'effet significatif sur l'usage de la contraception moderne. En revanche, l'instruction a un effet plus fort sur l'utilisation d'une contraception naturelle ou traditionnelle dans les quartiers les mieux équipés en infrastructures et en services $(P<0,01)$. Ainsi, dans ces quartiers dotés d'un meilleur niveau de développement, les femmes instruites ont 1,181 plus de chance d'utiliser une méthode naturelle ou traditionnelle que dans un quartier de faible niveau de développement. Il en est sensiblement de même dans les quartiers ayant bénéficié de campagnes de planification familiale, où les femmes instruites ont 1,105 plus de chance d'utiliser une méthode par rapport à celle résidant dans les quartiers qui n'en ont pas été dotés $(\mathrm{P}<0,05)$.

En milieu rural, de même qu'en zone urbaine, l'interaction entre l'instruction et le lieu de résidence a un effet significatif sur l'utilisation des méthodes contraceptives. Cet effet est plus significatif pour les méthodes naturelles et traditionnelles lorsque la femme réside dans les régions de savane que dans celles de forêt $(\mathrm{OR}=1,169$ et $\mathrm{p}<0,01)$. Par contre, si toutes les autres interactions observées indiquent un effet variable de l'instruction selon la nature du contexte, cet effet n'est pas significatif au seuil de 0,10 .

En définitive, on constate par cette analyse que l'effet de l'instruction varie significativement selon la région de résidence et l'on peut considérer que plus la région est globalement démunie, comme c'est le cas des régions de savane vis-à-vis de celles de forêt et plus encore vis à vis d'Abidjan, plus le progrès de l'instruction a un impact positif sur la pratique contraceptive. En revanche, lorsque l'on observe les interactions avec des facteurs qui 
\{PACFin Population Studies Vol. $21 n^{\circ} 1 /$ Etude de la population africaine vol. $21 n^{\circ} 2$

relèvent davantage de caractéristiques locales (niveau de développement, centres ou campagnes de planification familiale), l'impact de l'instruction sur la contraception ne varie pas significativement, surtout en milieu rural. 
TABLEAU 3. EFFET DES VARIABLES D'INTERACTION EN MILIEU URBAIN ET MILIEU RURAL. COTE D'IVOIRE. EDS 1994

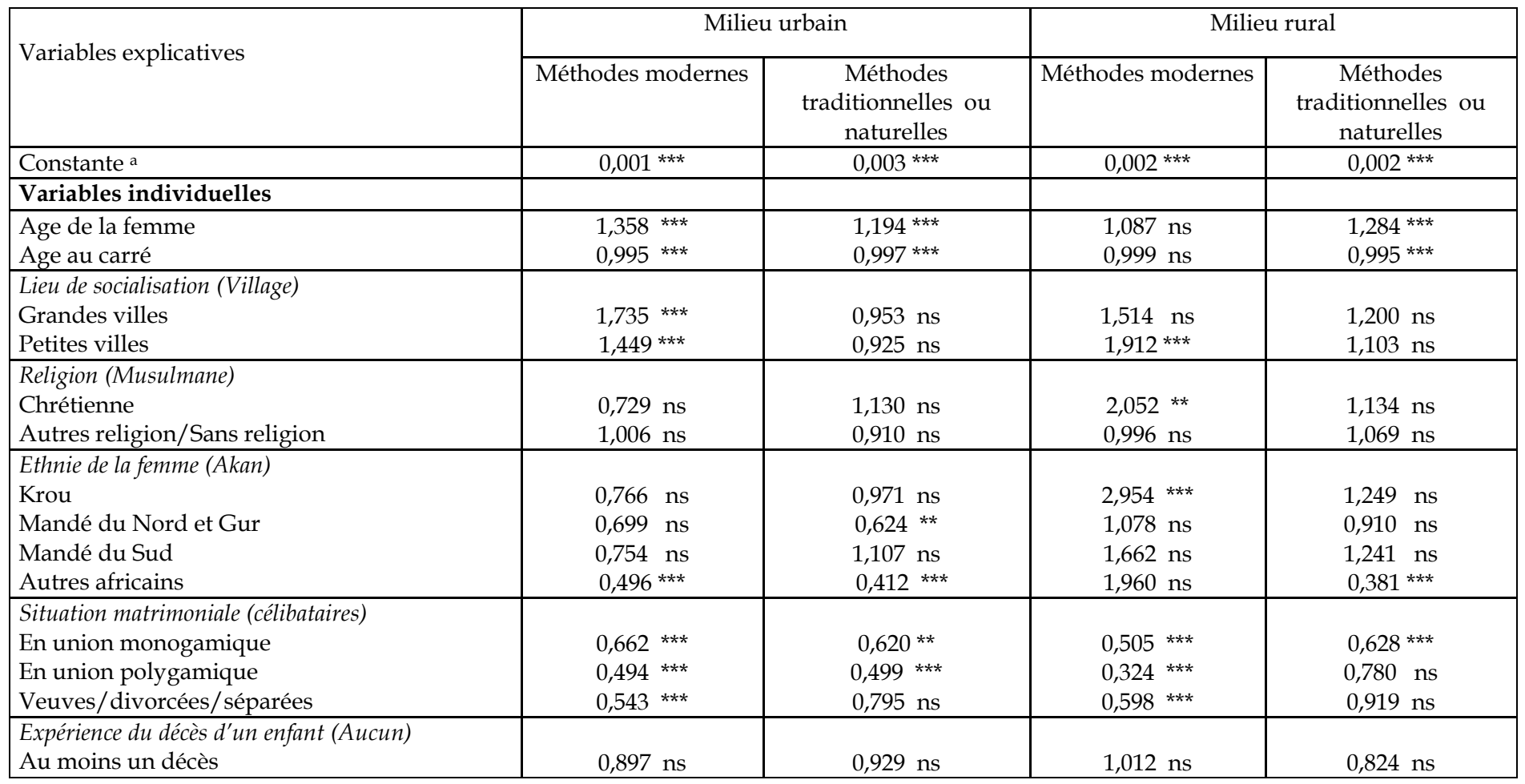


\{PAEFcal\} Population Studies Vol. $21 n^{\circ} 1 /$ Etude de la population africaine vol. $21 n^{\circ} 2$

\begin{tabular}{|c|c|c|c|c|}
\hline $\begin{array}{l}\text { Confiage des enfants (Au moins un enfant confié) } \\
\text { Aucun enfant confié }\end{array}$ & $0,555 * * *$ & $1,060 \mathrm{~ns}$ & $0,958 \mathrm{~ns}$ & $0,754 \mathrm{~ns}$ \\
\hline $\begin{array}{l}\text { Activité de la femme (Formel/informel) } \\
\text { Sans activité } \\
\text { Agricultrice }\end{array}$ & & & $\begin{array}{l}0,455 * * * \\
0,471 * * *\end{array}$ & $\begin{array}{c}1,071 \mathrm{~ns} \\
0,571 * * *\end{array}$ \\
\hline $\begin{array}{l}\text { Structure du ménage (Sans famille nucléaire) } \\
\text { Nucléaire monogame ou polygame } \\
\text { Élargie monogame } \\
\text { Élargie polygame }\end{array}$ & $\begin{array}{l}0,670 * * \\
0,847 \text { ns } \\
1,041 \text { ns }\end{array}$ & $\begin{array}{l}0,848 \mathrm{~ns} \\
0,945 \mathrm{~ns} \\
0,871 \mathrm{~ns}\end{array}$ & $\begin{array}{l}0,538 \mathrm{~ns} \\
0,929 \mathrm{~ns} \\
1,241 \mathrm{~ns}\end{array}$ & $\begin{array}{l}0,694 \mathrm{~ns} \\
0,980 \mathrm{~ns} \\
0,768 \mathrm{~ns}\end{array}$ \\
\hline $\begin{array}{l}\text { Niveau de vie du ménage (bas) } \\
\text { Élevé } \\
\text { Moyen }\end{array}$ & $\begin{array}{l}1,759 \mathrm{~ns} \\
1,085 \mathrm{~ns}\end{array}$ & $\begin{array}{l}0,613 * * \\
0,622 * *\end{array}$ & $\begin{array}{l}1,230 \mathrm{~ns} \\
0,726 \mathrm{~ns}\end{array}$ & $\begin{array}{l}0,724 \mathrm{~ns} \\
0,753 \mathrm{~ns} \\
\end{array}$ \\
\hline $\begin{array}{l}\text { \% d'utilisatrices de méthodes contraceptives } \\
\% \text { d'enfants scolarisés }\end{array}$ & $\begin{array}{l}1,026^{* * *} \\
1,004 \text { ns }\end{array}$ & $\begin{array}{l}1,053 * * * \\
0,986 * *\end{array}$ & $\begin{array}{l}1,030 * * \\
1,001 \mathrm{~ns} \\
\end{array}$ & $\begin{array}{l}1,092 * * * \\
0,976 * * *\end{array}$ \\
\hline
\end{tabular}




\begin{tabular}{|c|c|c|c|c|}
\hline \begin{tabular}{|l|} 
Variables d'interaction \\
\end{tabular} & & & & \\
\hline \begin{tabular}{|l|} 
Instruction / Région de résidence \\
Instruction / Niveau de développement \\
Instruction / Nombre de centres délivrant la \\
$\mathrm{PF}$ \\
Instruction / Campagne de PF \\
Instruction/ Mortalité des enfants \\
\end{tabular} & $\begin{array}{l}1,176 \text { ** } \\
1,027 \text { ns } \\
1,025 \text { ns } \\
1,060 \text { ns } \\
1,095 \text { ns }\end{array}$ & $\begin{array}{l}1,102 * * \\
1,181^{* * *} \\
0,975 \text { ns } \\
1,105 * * \\
1,055 \text { ns }\end{array}$ & $\begin{array}{l}1,158 \mathrm{~ns} \\
0,979 \mathrm{~ns} \\
0,933 \mathrm{~ns} \\
1,097 \mathrm{~ns} \\
0,936 \mathrm{~ns}\end{array}$ & $\begin{array}{l}1,169 \text { *** } \\
0,989 \text { ns } \\
1,035 \text { ns } \\
1,041 \text { ns } \\
0,965 \text { ns }\end{array}$ \\
\hline \begin{tabular}{|l|} 
Variance au niveau des grappes $\left(\sigma_{v}^{2}\right)$ \\
Variance au niveau des individus $\left(\sigma_{e}{ }^{2}\right)$
\end{tabular} & $\begin{array}{c}0,000 \\
1,000 * * *\end{array}$ & $\begin{array}{r}0,000 \\
1,000 * * * \\
\end{array}$ & $\begin{array}{c}0,000 \\
1,000 * * * \\
\end{array}$ & $\begin{array}{r}0,000 \\
1,000 * * *\end{array}$ \\
\hline
\end{tabular}


\{PACGia\} Population Studies Vol. $21 n^{\circ} 1 /$ Etude de la population africaine vol. $21 n^{\circ} 2$

\section{II.3. Limites de l'analyse}

La principale limite de l'analyse réside dans la façon dont la variable dépendante a été opérationnalisée. Deux sous variables dichotomiques ont été construites à partir des modalités de réponse d'une question générale sur la pratique actuelle de la contraception: l'utilisation actuelle des méthodes modernes et l'utilisation actuelle des méthodes traditionnelles. La modalité « oui $=1$ » pour la première sous variable concerne toutes les femmes qui utilisent une méthode de contraception moderne et la modalité " non $=0$ » concerne les autres cas. Ainsi, la modalité «non $=0$ » de la variable utilisation des méthodes modernes inclut également les femmes qui utilisent les méthodes traditionnelles. De même, la modalité «non $=0$ » de la variable utilisation des méthodes traditionnelles inclut à la fois les femmes qui n'utilisent aucune méthode et celles qui utilisent les méthodes modernes. Nous reconnaissons qu'une telle approche comporte un certain biais lié au mélange de deux catégories de populations: celles qui n'utilisent pas de méthodes de contraception et celles qui utilisent les méthodes alternatives. Cette façon de procéder est de nature à diminuer quelque peu les différences entre les catégories sociales et leur précision statistique. Il serait donc indiqué dans une étape ultérieure de recherche d'utiliser le modèle de régression logistique multinomial en construisant une variable dépendante avec trois modalités: (i) celles qui utilisent les méthodes modernes; (ii) celles qui utilisent les méthodes traditionnelles et (iii) celles qui n'utilisent aucune méthode contraceptive. Une autre limite de notre étude réside dans le fait que, la transition de la fécondité en étant à ses débuts en Côte d'Ivoire, le niveau de la pratique contraceptive est faible et les effets contextuels ne sont pas assez perceptibles.

\section{Conclusion}

Notre analyse a porté sur la relation entre les conditions de vie des femmes et leurs pratiques en matière de planification familiale, avec l'objectif de montrer comment les inégalités sociales, et plus particulièrement l'inégale répartition de la pauvreté des conditions d'existence, ont un impact significatif sur les pratiques en matière de contraception. Pour cela, nous avons distingué le milieu urbain du milieu rural en nous focalisant sur les femmes en union. Au niveau individuel, cette pauvreté a été mesurée par l'absence d'instruction, l'inactivité ou l'exercice d'une activité dans le secteur agricole ou informel et la précarité des conditions de vie dans le ménage. Au niveau contextuel, elle a été appréhendée par le manque d'accès à certaines infrastructures socioéconomiques modernes pour la satisfaction des besoins essentiels tels que l'électricité, l'eau potable, les centres de santé, les établissements scolaires, les routes, les équipements agricoles et les centres d'animation sociale et culturelle. Parce que nous disposions d'informations récentes sur le statut socio-économique de la femme et les caractéristiques de son milieu de résidence, nous avons jugé nécessaire de limiter l'analyse à l'utilisation d'une méthode contraceptive au moment de l'enquête en distinguant les méthodes modernes des méthodes naturelles ou traditionnelles. Le modèle multi niveau a été utilisé afin d'analyser l'influence relative de chacune des 
Edouard Talman \& Patrice Vimard: Développement local, pauvreté et pratiq|PAGE \} contraceptive en Côte d'Ivoire

variables retenues sur les décisions individuelles en matière de contraception. Nous avions posé l'hypothèse que de meilleures conditions de vie des femmes iraient de pair avec plus forte utilisation des méthodes contraceptives.

Les résultats que nous avons obtenus ont permis de confirmer cette hypothèse. Dans l'ensemble, ils restent conformes à ceux qui ont été obtenus par d'autres auteurs en montrant d'une part, que la pratique contraceptive, qu'elle soit moderne, naturelle ou traditionnelle, est plus répandue en milieu urbain qu'en zone rurale. D'autre part, l'usage de la contraception varie, à l'intérieur de chacune des zones de résidence, en fonction des caractéristiques socio-économiques individuelles et collectives, et l'on constate un très faible niveau d'utilisation des méthodes contraceptives dans les couches sociales défavorisées du point de vue de leur accès aux moyens d'instruction, de même que pour celles, souvent les mêmes, qui vivent dans des ménages et des contextes locaux dotés d'un faible niveau de vie. Tandis que dans les classes les plus aisées et instruites de la société urbaine, cette prévalence contraceptive est relativement élevée.

En ville les caractéristiques favorables à la pratique contraceptive concernent l'appartenance ethnique de la femme, son niveau d'instruction et le niveau de vie du ménage. Au plan contextuel, c'est le niveau de développement scolaire du quartier, dont les taux de scolarisation des enfants et des adultes sont des révélateurs, qui a un effet positif sur la prévalence contraceptive moderne. Ainsi en milieu urbain, le fait de vivre dans un ménage pauvre et un quartier dont la population est faiblement scolarisée conduit à un faible taux d'utilisation de la contraception moderne.

En milieu rural, l'instruction et l'activité dans les secteurs secondaire ou tertiaire, formel ou informel, ont un effet positif sur la pratique contraceptive. Les femmes illettrées ou qui vivent dans des ménages dont le chef est lui-même illettré sont moins susceptibles d'utiliser une contraception. Il en est de même des agricultrices et des femmes sans activité. À l'échelle contextuelle du village de résidence de la femme, aucune variable n'a d'effet sur l'utilisation des méthodes contraceptives modernes, mais deux d'entre elles ont un lien significatif avec l'utilisation des méthodes naturelles ou traditionnelles : le niveau de développement socio-économique du village et le niveau de la mortalité des enfants de moins de cinq ans. En milieu rural, la pauvreté, associée aux conditions de vie les plus basses et à la plus forte mortalité des enfants, correspond par conséquent à la plus faible prévalence de la contraception naturelle ou traditionnelle et se trouve donc associée à une moindre régulation de la fécondité.

Si l'instruction joue en elle-même un rôle très important, voire majeur, sur la propension à utiliser une contraception, notre analyse des interactions a montré qu'elle n'avait pas de véritable effet interactif avec les variables du développement local que nous avons identifiées. Par contre son effet sur l'augmentation de la contraception est plus important dans les régions de savanes, correspondant à la moitié nord du pays et à la zone la plus pauvre de Côte d'Ivoire. 
\{PASE

L'ensemble de nos résultats confirme l'association de la pauvreté humaine, caractérisée dans notre étude par un faible niveau d'instruction et des conditions de vie familiales et locales précaires, avec la faible pratique contraceptive. Il indique tout l'effort qui reste à faire au niveau politique et institutionnel pour étendre l'accès à l'éducation à toutes les couches sociales et améliorer les conditions de vie des populations. En attendant que l'instruction et l'insertion des femmes dans les activités secondaires et tertiaires se généralisent, des efforts doivent être entrepris pour faciliter l'accès à la planification familiale par les groupes confrontés à des problèmes et à des obstacles particuliers, tels les analphabètes et les pauvres des zones rurales qui sont pour la plupart très éloignés des centres de santé où se délivrent généralement ces services (Vimard et al., 2002). Le gouvernement et les prestataires des services de planification familiale ont la responsabilité de s'assurer que les interventions visant à accroitre l'usage des méthodes contraceptives sont de qualité et qu'elles prennent suffisamment en compte les préoccupations des catégories vulnérables de la population. Ils devraient aussi travailler avec ces individus de manière à accroître leurs connaissances en leur fournissant des informations pertinentes et objectives sur les propriétés des différentes méthodes contraceptives en particulier celles des méthodes modernes. Ils devraient chercher à accroître le soutien dont pourraient bénéficier les femmes en prenant en compte les préoccupations des hommes en matière de planification familiale. Car, il semble que les hommes ont besoin d'être motivés à l'utilisation des méthodes contraceptives par les femmes. Il est clair que notre analyse ne prend pas en compte toute la réalité socioculturelle qui entoure la planification familiale dans le pays. Il importe donc d'approfondir cette étude en intégrant des variables relatives aux normes, aux contraintes économiques et au coût des méthodes contraceptives. 
Edouard Talman \& Patrice Vimard: Développement local, pauvreté et pratiq|PAGE \} contraceptive en Côte d'Ivoire

Note :

1) Pour une approche plus complète de l'évolution de la fécondité en Côte d'Ivoire, à l'aide des modèles multi niveaux, on pourra se reporter à Talnan, 2005.

2) L'enquête démographique et de santé de 1994 a été choisie de préférence à celle de 1998-99 car son échantillon est plus important et ses données apparaissent de meilleure qualité.

3) Bien que traduisant le contexte socioculturel de la femme, l'ethnie, la religion et le lieu de socialisation sont utilisés ici comme des variables individuelles pour la simple raison qu'en Côte d'Ivoire, il n'est pas rare de trouver plusieurs femmes d'ethnies ou de religion différentes cohabiter dans un même lieu de résidence à cause des migrations internes et internationales. C'est ainsi qu'une femme peut se retrouver dans une localité où l'ethnie et la religion dominantes ne sont pas les siennes. Elle pourrait avoir tendance à se conformer aux règles prônées par sa religion ou son ethnie pour ce qui est des pratiques reproductives surtout lorsqu' elle n'a pas été socialisée dans ce lieu de résidence. En ce sens, ces variables agissent comme des caractères individuels plutôt que collectif.

4) La structure familiale du ménage de résidence est construite à partir du lien de parenté avec le chef de ménage. Elle comportait au départ sept modalités : les ménages sans famille nucléaire composés du chef de ménage et de ses enfants, les ménages monoparentaux simples composés d'un des parents et de ses enfants sans la présence d'une autre personne, les ménages monoparentaux élargis à d'autres personnes apparentées ou non au chef de ménage, les ménages nucléaires monogames ou polygames et les ménages élargis monogames ou polygames. Mais compte tenu de la rareté statistique qui caractérisait certaines modalités, nous avons procédé à des regroupements pour aboutir à quatre modalités qui sont: les ménages sans famille nucléaire composés des ménages où le chef est seul sans épouses ni enfants, et des ménages monoparentaux (simples ou élargis); les ménages nucléaires monogames ou polygames; les ménages monogames élargis; les ménages polygames élargis.

5) Sur la base de cette classification, $17,8 \%$ des femmes de notre échantillon $\mathrm{n}^{\prime}$ ont aucun accès aux services de planification familiale en milieu urbain, $39,1 \%$ ont un accès très difficile, $24,7 \%$ ont un accès difficile, $10,7 \%$ ont un accès facile et 7,7 \% ont un accès très facile. Dans les zones rurales, 13,9\% des femmes n'ont pas du tout accès à ces services, $42,3 \%$ y ont accès très difficilement, $25 \%$ y ont accès difficilement, alors que $13,7 \%$ y ont accès facilement et $4,9 \%$ ont un accès très facile à ces services de planification familiale.

6) L'utilisation d'un modèle vide permet de mieux analyser les variations de la variance contextuelle au fur et à mesure que l'on introduit des variables explicatives dans le modèle.

7) Certaines variables telles que l'accessibilité à la planification familiale, le nombre de noyaux familiaux présents dans le ménage autres que celui constitué par le chef de ménage et l'expérience individuelle du décès d'un 
enfant ont été exclues de l'analyse parce qu'elles n'ont pas montré de liens significatifs avec la pratique contraceptive en milieu urbain comme dans les zones rurales.

\section{Références :}

Alaudin M., 1983, «Community factors in contraception among Bangladeshi Rural Women ", in Casterline J.B. (éd.), WFS Seminar on the collection and Analysis of Data on Community and Institutional Factors, 20-23 June 1983. pp. 3-13.

Anoh A. Fassassi R. et Vimard P., 2005, «Politique de population et planification familiale en Côte d'Ivoire ", in Gautier A. (éd.), Les politiques de planification familiale. Cinq expériences nationales, CEPED-LPED-INED, Paris, pp. 195-231.

Ainsworth M., Beegle K. and Nyamete A., 1996, « The impact of women's schooling on fertility and contraceptive use : A study of fourteen sub-saharan African countries ", The World Bank economic Review, volume 10, Number 2, pp. 85122.

Bahattacharyya Amit K., 1989, « An evaluation of the impact of development projects on fertility: Experience from developing countries", Communication au XXIe Congrès général de la population, UIESP, New Delhi, 1989, vol. 2, pp. 305-315.

Benefo K. and Shultz T. P, 1996, «Fertility and child mortality in Côte d'Ivoire and Ghana », The World Bank economic Review, volume 10, Number 2, pp.123-157.

Bilsborrow R., Hess P. and Tsui A., 1989, "Linkage between, the demand for children, contraceptive use, and fertility: preliminary evidence from Mexico and unresolved issues », Communication au XXIe Congrès général de la population, UIESP, New Delhi, 1989, vol. 1, pp. 331-348.

Casterline J.B, 1985, «Community effects on fertility », in Casterline J. B. (ed.), The Collection and Analysis of Community Data, (Voorburg, Netherlands: International Statistical Institute), pp. 65-75.

De Jong G. F., Robinson W. C., Hoque N. M. and Cornwell G. T., 1989, «Rural electrification and fertility attitudes and behaviour in Bangladesh", Communication au XXIe Congrès général de la population, UIESP, New Delhi, 1989, Vol. 1, pp. 317-328.

Entwisle B., Mason W.M. and Hermalin A.I., 1986, « The multilevel dependence of contraceptive use on socio-economic development and family planning program strength », Demography, Vol. 23, Number 2, pp. 199-216.

Guilkey D. K. and Jayne S., 1997, « Fertility transition in Zimbabwe: Determinants of contraceptive use and method choice », Population Studies, Vol. 51, pp. 153189.

INS (Institut national de la statistique) et ORC Macro, 2001, Enquête démographique et de santé. Côte d'Ivoire 1998-1999, Institut National de la Statistique - ORC Macro, Abidjan et Calverton, 298 p.

Kalam E.M.N and Khan H.T.A, 1996, Modeling contraceptive prevalence in Bangladesh: A hierarchical Approach, Asian metacentre research paper series, no 5, $24 \mathrm{p}$. 
Edouard Talman \& Patrice Vimard: Développement local, pauvreté et pratiqPAGE \} contraceptive en Côte d'Ivoire

Kouamé Aka, Schelllekens Jona, 2002, Développement rural et attitude à l'égard de la taille de la famille en Côte d'Ivoire, Population, Numéro 2, volume 57, 293-326, MarsAvril 2002.

Mancini D. J., Stecklov G. and Stewart J. F., 2003, "The effect of structural characteristics on family planning program performance in Côte d'Ivoire and Nigeria ", Social Science and Medecine, 56 (2003), pp. 2123-2137.

Nguyen M. et Nguyen A, 2002, «Acceptability and use of contraceptives in Vietnam », International Family Planning Perspectives, 28 (4), pp. 214-219.

Schoumaker B., 2001a, «Une analyse multiniveaux dynamique de la fécondité légitime au Maroc rural », Communication au XXIVe Congrès général de la population, UIESP, Salvador de Bahia, Brésil, 18-24 Août 2001, 25 p.

Schoumaker B., 2001b, "Analyse multi-niveaux des déterminants de la fécondité : Théories, méthodes et applications au Maroc rural », Thèse de doctorat, 339 p.

Snijders T.A.B and Bosker R.J, 1999, Multilevel Analysis : An introduction to basic and advanced multilevel modelling, Sage Publications, London, Thousand Oaks, New delhi, $260 \mathrm{p}$.

Vimard P., Anoh A. et Guillaume A, 2002, "La croissance démographique », in Tapinos G.P., Hugon P. et Vimard P. (éds), La Côte d'Ivoire à l'aube du 21e siècle: défis démographiques et développement durable, Edition Karthala, 2002, pp.15-87. 